\section{A) Check for updates}

Cite this: Food Funct., 2022, 13, 1246

\title{
The garlic-derived organosulfur compound diallyl trisulphide suppresses tissue factor function
}

\author{
Sachiko Okue, ${ }^{a}$ Manami Yaguchi, ${ }^{a}$ Atsushi Miura, ${ }^{a}$ Yori Ozaki-Masuzawa, ${ }^{\text {b }}$ \\ Takashi Hosono (iD ${ }^{a, b}$ and Taiichiro Seki (D) *a,b
}

\begin{abstract}
Tissue factor (TF) is a critical initiator of extrinsic coagulation that sometimes causes thromboembolism. Diallyl trisulphide (DATS) is a secondary metabolite of allicin generated in crushed garlic, with various pharmacological effects. This study aimed to clarify the effect of DATS on the extrinsic coagulation elicited by TF and arteriosclerosis. TF activity was measured using a clotting assay in TF-expressing HL60 cells. DATS inhibited TF activity in a dose-dependent manner. TF expression in TNF- $\alpha$-stimulated human umbilical vein endothelial cells was examined using real-time PCR and western blotting. DATS inhibited TF mRNA and protein expression induced by TNF- $\alpha$ via inhibition of JNK signalling. The effect of DATS on arteriosclerosis was also examined in apolipoprotein E-deficient mice. DATS administration in these mice tended to decrease atherosclerotic lesion size. These results strongly suggest that DATS prevents thromboembolism triggered by atherosclerosis via the inhibition of plaque formation and TF function.
\end{abstract}

Received 11th July 2021,

Accepted 27th December 2021

DOI: $10.1039 / \mathrm{d} 1 \mathrm{fo} 02206 \mathrm{~g}$

rsc.li/food-function particles released from atherosclerotic plaques, which subsequently promote thrombus formation. ${ }^{14-16}$

There are two types of TF: activated and inactivated forms. Changes in cell structure due to plasma membrane damage, apoptosis or exposure to oxidising agents ${ }^{17}$ and calcium ionophores $^{18}$ induce transformation of inactivated TF to its active form. It has previously been reported that the formation of a disulphide bond between $\mathrm{Cys}_{186}$ and $\mathrm{Cys}_{209}$ in the TF molecule is important for its activity. ${ }^{18,19}$

Garlic (Allium sativum L.) is one of the first plants used for medicinal purposes. It is also used as a spice and food. ${ }^{20}$ Garlic exerts various pharmacological effects, including antihypertensive, ${ }^{21,22}$ anti-obesity, ${ }^{23-25}$ anti-thrombogenic and anti-platelet aggregation ${ }^{26-28}$ activities. However, the mechanisms underlying these effects have not been clearly elucidated.

In crushed and cooked garlic, the volatile organosulphur compound allicin is generated by alliinase enzymatic activity. Allicin is converted into several sulphide compounds, including DATS, diallyl disulphide, diallyl monosulphide and methyl allyl trisulphide, by spontaneous non-enzymatic reactions. ${ }^{29}$ These organosulphur compounds are presumably responsible for the physiological effects of garlic. Indeed, we recently reported inhibition of platelet aggregation by DATS. ${ }^{28}$ We demonstrated that DATS suppresses integrin $\alpha \operatorname{IIb} \beta 3$ activation and the release of $\alpha$-granules via sulphhydryl groups on platelets. ${ }^{28}$ Platelets play important roles in haemostasis, development of arteriosclerosis and the pathogenesis of cardiovascular diseases. Indeed, platelets tend to aggregate around atherosclerotic lesions, triggering thrombus formation. ${ }^{30}$

\footnotetext{
${ }^{a}$ Department of Applied Life Sciences, Nihon University Graduate School of Bioresource Sciences, Kanagawa, Japan. E-mail: seki.taiichirou@nihon-u.ac.jp ${ }^{b}$ Department of Chemistry and Life Science, Collage of Bioresource Sciences, Nihon University, Kanagawa, Japan
} 
This study was performed to determine the effects of DATS on the development of arteriosclerosis and thrombus formation triggered by the extrinsic coagulation pathway to further understand the properties of garlic in the prevention of cardiovascular diseases. First, we investigated the effects of DATS on the expression and activity of TF, which plays a crucial role in the extrinsic coagulation cascade, leading to thrombotic disease. Second, we examined the effects of DATS on the development of atherosclerosis in apolipoprotein $\mathrm{E}$ (ApoE)-deficient mice.

\section{Materials \& methods}

\subsection{Chemicals, reagents and antibodies}

DATS was synthesised using Bunte salts as described previously. ${ }^{28}$ DATS was further purified by high-performance liquid chromatography (Alliance 2695; Waters, Milford, MA, USA) on an Inertsil ODS-3 column $(6 \times 250 \mathrm{~mm}$; GL Science, Tokyo, Japan), achieving a purity of $99 \%$. Other chemicals, including $N$-ethylmaleimide (NEM), L-glutamine, PMA, dimethyl sulphoxide (DMSO), medium 199 and heparin sodium salt, were obtained from Sigma-Aldrich (St Louis, MO, USA). Roswell Park Memorial Institute 1640 medium (RPMI 1640 medium) and Alexa Fluor 488-conjugated donkey antirabbit IgG $(\mathrm{H}+\mathrm{L})$ highly cross-adsorbed secondary antibody, were purchased from Thermo Fisher Scientific (Waltham, MA, USA). Endothelial Cell Basal Medium 2 (EBM-2) and Supplement Pack Endothelial Cell GM 2 were purchased from Promo Cell (Heidelberg, Germany). Foetal bovine serum (FBS) was purchased from Biowest (Nuaillé, France). Recombinant human basic fibroblast growth factor was purchased from Aviva Systems Biology (San Diego, CA, USA). RNAiso Plus kit and PrimeScript RT Reagent kit were purchased from Takara Bio (Shiga, Japan). Rabbit anti-TF antibody was purchased from Santa Cruz Biotechnology (Dallas, TX, USA). Rabbit antiphospho-SAPK/JNK (Thr183/Tyr185) antibody, rabbit antiJNK2 monoclonal antibody and rabbit anti-phospho-c-Jun (Ser63) antibody were purchased from Cell Signaling Technology (Danvers, MA, USA). Mouse anti- $\beta$-actin antibody was purchased from Sigma-Aldrich and horseradish peroxidase-conjugated secondary antibodies were purchased from Jackson ImmunoResearch (West Grove, PA, USA). 4',6Diamidino-2-phenylindole (DAPI) was purchased from Dojindo Laboratories (Kumamoto, Japan).

\subsection{Cell culture}

The human promyelocytic leukaemia cell line HL60 (cell no. RCB0041, Riken, Ibaraki, Japan) was maintained in RPMI 1640 medium supplemented with 10\% FBS. Human umbilical vein endothelial cells (HUVECs) (cell no. 03331; Kurabo, Osaka, Japan) were maintained in medium 199 supplemented with $20 \% \mathrm{FBS}, 2.5 \mathrm{ng} \mathrm{\textrm {mL } ^ { - 1 }}$ recombinant human basic fibroblast growth factor, $10 \mathrm{U} \mathrm{mL}^{-1}$ heparin sodium salt and $0.2 \mathrm{mg} \mathrm{mL}{ }^{-1}$ L-glutamine. For the experiments shown in Fig. 2 (D and E) and 4, HUVECs (Promo Cell) were maintained in
EBM-2 supplemented with 10\% FBS and Supplement Pack Endothelial Cell GM 2. Both HL60 cells and HUVECs were cultured at $37{ }^{\circ} \mathrm{C}$ in $5 \% \mathrm{CO}_{2}$ and $95 \%$ air.

\subsection{Clotting assay}

We performed clotting assays to determine the effects of DATS on TF activity using bovine plasma and human promyelocytic leukaemia HL60 cells. The clotting assay in HL60 cells was performed as described previously with minor modifications. ${ }^{18}$ Briefly, HL60 cells were plated at $1.0 \times 10^{6}$ cells per $\mathrm{mL}$ in RPMI 1640 medium supplemented with 10\% FBS and cultured for $24 \mathrm{~h}$. PMA solution in DMSO was then added to the culture to a final concentration of $1 \mu \mathrm{M}$, and the cells were incubated for $6 \mathrm{~h}$ to induce TF expression. After induction of TF by PMA, the cells were washed twice in 2-[4-(2-hydroxyethyl)piperazin-1yl] ethane sulphonic acid (HEPES)-buffered saline (10 mM HEPES, $137 \mathrm{mM} \mathrm{NaCl}, 5.38 \mathrm{mM} \mathrm{KCl}$ and $5.55 \mathrm{mM}$ glucose) and suspended in HEPES-buffered saline at $1.0 \times 10^{6}$ cells per $\mathrm{mL}$. This HL60 cell suspension $(200 \mu \mathrm{L})$ was pre-incubated at $37^{\circ} \mathrm{C}$ for $3 \mathrm{~min}$ and then treated with DATS $(40,80,120,160$ and $200 \mu \mathrm{M})$ and NEM $(40,80,120,160$ and $200 \mu \mathrm{M})$ (treatment group) or DMSO (vehicle control group) at $37{ }^{\circ} \mathrm{C}$ for $5 \mathrm{~min}$. The HL60 cells were then incubated with $100 \mu \mathrm{M} \mathrm{HgCl}_{2}$ for $30 \mathrm{~s}$ to activate TF. Bovine plasma $(50 \%(\mathrm{v} / \mathrm{v})$ in phosphate buffered saline (PBS), $100 \mu \mathrm{L}$ ) was added to the cell suspension mixture, and TF activity was measured as a function of clotting time.

\subsection{Western blotting}

HUVECs were grown to confluence, and the medium was replaced with fresh 199 medium or fresh EBM-2 supplemented with $2 \%$ FBS. DATS or DMSO was added to the culture medium and incubated for $30 \mathrm{~min}$. After pre-incubation with DATS or DMSO, TNF- $\alpha\left(1 \mathrm{ng} \mathrm{mL}{ }^{-1}\right)$ was added to the cells for various times. The cells were subsequently harvested in $50 \mathrm{mM}$ Tris-HCl (pH 8.0), $150 \mathrm{mM} \mathrm{NaCl}, 0.1 \%$ SDS, $0.5 \%$ deoxycholic acid and $1 \%$ NP-40. The cell lysates were subjected to sodium dodecyl sulphate-polyacrylamide gel electrophoresis $(10 \%$ gel), followed by transfer onto polyvinylidene difluoride membranes (Immobilon-P; Merck KGaA, Darmstadt, Germany) for western blotting. The blots were incubated with rabbit anti-TF antibody (1:1000), rabbit anti-phospho-SAPK/JNK (Thr183/ Tyr185) antibody (1:1000), rabbit anti-JNK2 monoclonal antibody $(1: 1000)$ or mouse anti- $\beta$-actin antibody $(1: 10000)$ at $4{ }^{\circ} \mathrm{C}$ overnight. After incubation with horseradish peroxidaseconjugated secondary antibody $(1: 20000)$ for $30 \mathrm{~min}$, the antigenic protein on the membrane was visualised by ImmunoStar LD (Fujifilm Wako Pure Chemical Co., Osaka, Japan) using the ChemiDoc MP Imaging System (Bio-Rad Laboratories, Hercules, CA, USA) for chemiluminescence detection.

\subsection{Reverse transcription and real-time PCR}

HUVECs were grown to confluence, and the medium was replaced with fresh 199 medium or fresh EBM-2 supplemented with $2 \%$ FBS. DATS or DMSO was added to the culture medium and incubated for $30 \mathrm{~min}$. After pre-incubation with 
DATS or DMSO, TNF- $\alpha\left(1 \mu \mathrm{g} \mathrm{mL} \mathrm{m}^{-1}\right)$ was added to the cells and incubated for various times. Total RNA was then extracted using the RNAiso Plus kit, and cDNA was synthesised using the PrimeScript RT Reagent kit. Real-time reverse-transcription PCR was performed using the KOD SYBR qPCR Mix (Toyobo Co., Osaka, Japan) on the StepOne PCR system (Thermo Fisher Scientific). The following human TF-, Icam-1- and Vcam-1specific primers used in this study were designed based on GenBank information and synthesised by Thermo Fisher Scientific: human TF, 5'-CCCCAGAGTTCACACCTTACCT-3' and 5'-CACTTTTGTTCCCACCTGTTCA-3'; human Icam-1 5'-ATGCCCAGACATCTGTGTCC-3' and 5'-GGGGTCTCTATGCCCAACAA-3'; human Vcam-1 5'-TTTGACAGGCTGGAGATAGACT-3' and 5'TCAATGTGTAATTTAGCTCGGCA-3'. mRNA levels were quantified using the $2^{-\Delta \Delta \mathrm{CT}}$ method $^{31}$ and adjusted relative to those of GAPDH, which was amplified using the primers $5^{\prime}$ CACCACCCTGTTGCTGTA- ${ }^{\prime}$ ' and 5'-CCACTCCTCCACCTTTGA$3^{\prime}$, as reported previously. ${ }^{31}$

\subsection{Immunofluorescence}

HUVECs were grown to confluence, and the medium was replaced with fresh EBM-2 supplemented with 2\% FBS. DATS or DMSO was added to the culture medium and incubated for $30 \mathrm{~min}$. After pre-incubation with DATS or DMSO, TNF- $\alpha(1 \mu \mathrm{g}$ $\mathrm{mL}^{-1}$ ) was added to the cells for $15 \mathrm{~min}$. The cells were fixed with acetone: methanol $(1: 1)$ and blocked with 10\% donkey serum in PBS. The cells were incubated with rabbit antiphospho-c-Jun (Ser63) antibody $(1: 400)$ for $1 \mathrm{~h}$, followed by Alexa Fluor 488-conjugated donkey anti-rabbit IgG $(\mathrm{H}+\mathrm{L})$ cross-adsorbed secondary antibody $(1: 500)$ for $30 \mathrm{~min}$. The cells were then treated with DAPI $(1: 1000)$ to stain the nuclei. Images were obtained by confocal microscopy (TCS SP8 LIGHTNING; Leica, Wetzlar, Germany).

\subsection{Mice and diets}

All experiments were performed in accordance with the National Institutes of Health Guide for the Care and Use of Laboratory Animals and were approved by the Nihon University Animal Care and Use Committee (approval number AP18BRS082-3). The animals used in this study were 6-week-

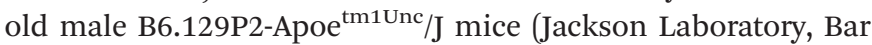
Harbor, ME, USA). Animals were housed in a temperature-controlled room $\left(22^{\circ} \mathrm{C}-23^{\circ} \mathrm{C}\right)$ under a $12 \mathrm{~h}$ light/dark cycle. The mice were provided a normal chow diet (CRF-1; Oriental Yeast Co., Tokyo, Japan) and water ad libitum. After 2 weeks of acclimation, the mice were divided into vehicle control and DATS groups matched for body weight. DATS was administered by oral gavage every other day for 12 weeks $(25 \mu \mathrm{mol}$ per $\mathrm{kg}$ body weight during the first week, $50 \mu \mathrm{mol}$ per $\mathrm{kg}$ body weight during the second week and $75 \mu \mathrm{mol}$ per kg body weight thereafter), because DATS has a characteristic flavour that sometimes influences food intake. The dose of DATS was increased gradually to acclimatise the mice to its smell. The same amount of solvent $(99.5 \%$ saline, $0.5 \%$ polyoxyethylene (20) sorbitan monooleate) without garlic DATS was administered to the control group.

\subsection{Histology}

Mice were euthanised by carbon dioxide inhalation after 12 weeks of DATS administration. Harvested common carotid arteries were fixed using $4 \%$ paraformaldehyde. The tissue samples were embedded in optimal cutting temperature (OCT) compound, and axial sections $10 \mu \mathrm{m}$ thick were cut using a cryostat microtome (CM 3050 S; Leica). Serial cryosections of the tricuspid valve were analysed. The sections were stained with haematoxylin and eosin for plaque morphology and Oil Red $\mathrm{O}$ for lipid deposition, and staining was evaluated using an upright light microscope (Axio Imager A2; Carl Zeiss, Oberkochen, Germany). The lesion areas of each serial section were measured using ImageJ (version 1.52k; National Institutes of Health, Bethesda, MD, USA).

\subsection{Statistical analysis}

All data are expressed as the mean \pm standard error and were analysed using Prism 8 software (GraphPad Software, San Diego, CA, USA). Statistical comparisons between the two groups were performed using Student's $t$ test. One-way analysis of variance (ANOVA) was used for multiple comparisons, and Dunnett's test was used to analyse group differences. Two-way ANOVA was used for examination of the interaction between DATS treatment and the time after treatment with TNF- $\alpha$, and the Tukey-Kramer test was used to analyse group differences. In all analyses, $P<0.05$ was taken to indicate statistical significance.

\section{Results}

\subsection{DATS prolongs clot formation}

In this study, a clotting assay system was used to determine the effects of DATS on TF activity. In the body, TF is activated by protein disulphide isomerase, ${ }^{19}$ which forms a disulphide bond between $\mathrm{Cys}_{186}$ and $\mathrm{Cys}_{209}$, resulting in TF activation. In the clotting assay, PMA-stimulated HL60 cells were further treated with $\mathrm{HgCl}_{2}$ to activate TF. In vehicle-treated HL60 cells (without PMA), $\mathrm{HgCl}_{2}$ treatment did not affect clotting time (Fig. 1A). In contrast, clotting time was significantly decreased by $\mathrm{HgCl}_{2}$ treatment in PMA-stimulated, TF-expressing HL60 cells (Fig. 1A). These data suggest that TF expression in HL60 cells is activated by disulphide bond formation.

We next examined the effects of DATS on clot formation elicited by TF (Fig. 1B). DATS was added to PMA-stimulated, TFexpressing HL60 cells followed by activation with $\mathrm{HgCl}_{2}$. As shown in Fig. 1B, DATS delayed clotting time in a dose-dependent manner. The effect of the SH group modifier, NEM, was also examined. Treatment with NEM significantly inhibited clot formation in a manner similar to DATS (Fig. 1C).

\subsection{DATS suppresses TF expression in vascular endothelial cells}

TF expression in vascular endothelial cells is induced by inflammation, which is a cause of thrombosis during atherosclerosis. ${ }^{14,32}$ We used confluent HUVECs to determine 


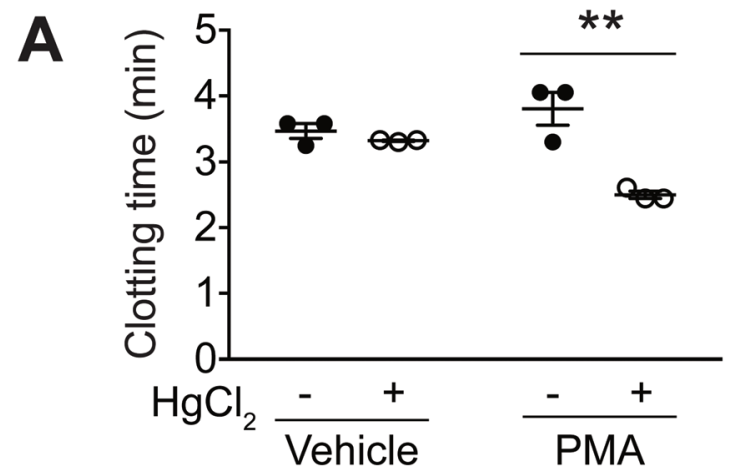

B
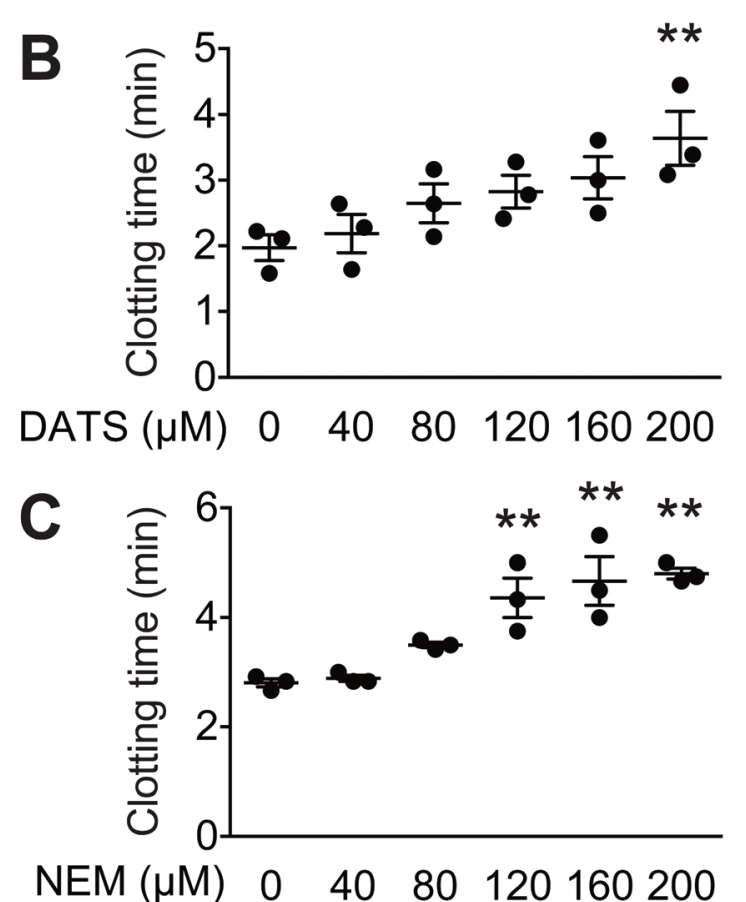

Fig. 1 DATS inhibits TF activity and subsequent clot formation. (A) PMA-stimulated, TF-expressing HL60 cells and vehicle-treated, non-TFexpressing $\mathrm{HL} 60$ cells were further treated with $\mathrm{HgCl}_{2}$ to activate TF. Bovine plasma was added to these cells to measure TF activity as clotting time. (B) DATS was added to the $\mathrm{PMA} / \mathrm{HgCl}_{2}$-treated cells $5 \mathrm{~min}$ prior to measuring clotting time. (C) NEM was added to the $\mathrm{PMA} / \mathrm{HgCl}_{2}-$ treated cells 5 min prior to measuring clotting time. Mean \pm standard error (SE) $(n=3),{ }^{*} P<0.05$, ${ }^{*} P<0.01$ vs. (A) $\mathrm{HgCl}_{2}(-)$, (B and C) $0 \mu \mathrm{M}$.

the effects of DATS on TF expression in vascular endothelial cells; thus, cell number and proliferation did not influence the results obtained in this study. Incubation of HUVECs with TNF- $\alpha$ resulted in induction of TF mRNA expression, which peaked at $2 \mathrm{~h}$ (Fig. 2A, black bars). TF mRNA induction by TNF- $\alpha$ was significantly suppressed by DATS (Fig. 2A, grey bars). Similarly, TF protein expression in HUVECs was induced by TNF- $\alpha$, peaking at $4 \mathrm{~h}$ after treatment (Fig. 2B). DATS also inhibited TF protein induction by TNF- $\alpha$ in a dose-dependent manner (Fig. 2C). Next the phosphorylation of JNK1/2 by TNF$\alpha$ was measured to explore the inhibitory mechanisms of DATS on TF induction. JNK was transiently phosphorylated by TNF- $\alpha$ by 15 min in HUVECs, and DATS inhibited TNF- $\alpha$-induced phosphorylation of JNK (Fig. 2D). Moreover, we examined the effects of DATS on nuclear localisation of phosphorylated c-Jun, a downstream substrate of the JNK signalling pathway. Phosphorylated c-Jun was localised in nuclei by TNF- $\alpha$ at $15 \mathrm{~min}$, and pre-treatment with DATS inhibited TNF- $\alpha$-induced nuclear localisation of phosphorylated c-Jun (Fig. 2E). These results suggested that DATS suppresses TF expression via the inhibition of JNK signalling induced by TNF- $\alpha$.

\subsection{DATS ameliorates plaque formation in ApoE-deficient mice}

As described above, DATS prolonged clotting time by suppressing TF activity as well as TNF- $\alpha$-induced TF mRNA and protein expression. We next examined the effects of DATS on arteriosclerosis in ApoE-deficient mice, which are widely used as a model of human atherosclerosis (Fig. 3). ApoE is one of the major apolipoproteins in plasma lipoproteins, such as lowdensity lipoprotein and high-density lipoprotein. ApoE is responsible for the transport of lipids, including cholesterol. ${ }^{33}$ There were no significant differences in body weight (Fig. 3A), or plasma triglyceride (Fig. 3B) or cholesterol concentrations (Fig. 3C) between the vehicle group and DATS group. In vehicle-treated ApoE-deficient mice, mild plaque formation in the tricuspid valve was observed (Fig. 3D and E). In contrast, intraperitoneal injection of DATS $\left(75 \mu \mathrm{mol} \mathrm{kg}{ }^{-1}\right.$ body weight) once every 2 days tended to suppress this plaque formation in ApoE-deficient mice (Fig. 3D). The evaluation of atherosclerotic lesion size was performed using Oil Red $\mathrm{O}$ staining of serial tricuspid valve sections. Plaque formation in the tricuspid valve tended to be suppressed in the DATS treatment group compared with the vehicle group (Fig. 3D), although this effect was not statistically significant (Fig. 3E). These data suggest that DATS suppresses the onset of arteriosclerosis.

\subsection{DATS suppresses adhesion molecule expression in vascular endothelial cells}

During the formation of atherosclerotic plaque, adhesion of leukocytes and migration thereof into the vascular subendothelium are induced by Icam-1 and Vcam-1 in activated endothelial cells. As DATS ameliorated plaque formation in ApoE-deficient mice (Fig. 3), we postulated that DATS may regulate adhesion molecule expression in vascular endothelial cells during plaque formation. Therefore, we next evaluated the effects of DATS on Icam-1 and Vcam-1 mRNA expression in TNF- $\alpha$-stimulated HUVECs. The levels of Icam- 1 and Vcam1 mRNA expression were significantly increased $2 \mathrm{~h}$ after stimulation with TNF- $\alpha$, and DATS suppressed these TNF$\alpha$-induced increases in adhesion molecule mRNA expression (Fig. 4). These results suggested that DATS suppresses plaque formation by inhibiting the expression of adhesion molecules in vascular endothelial cells. 
A

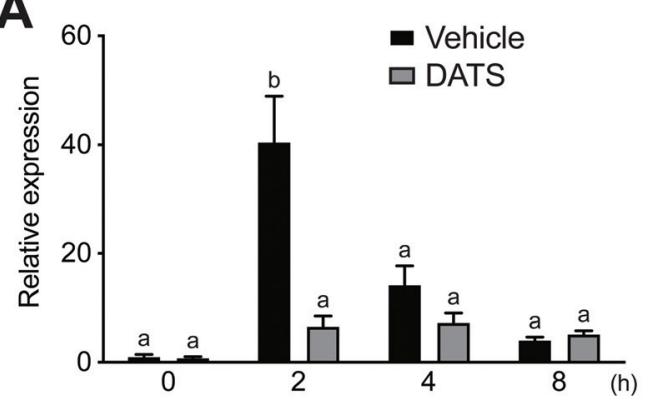

B After TNF- $\alpha$ stimulation (h)

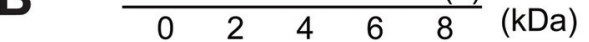

$$
\text { TF }
$$

$\beta$-actin
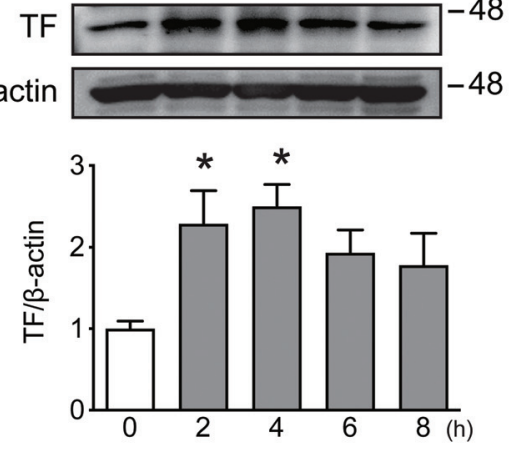

C

TNF- $\alpha(1 \mathrm{ng} / \mathrm{mL}) \quad-\quad+\quad+\quad+$

DATS $(\mu \mathrm{M}) \quad-\quad$ - $50 \quad 100 \quad(k D a)$
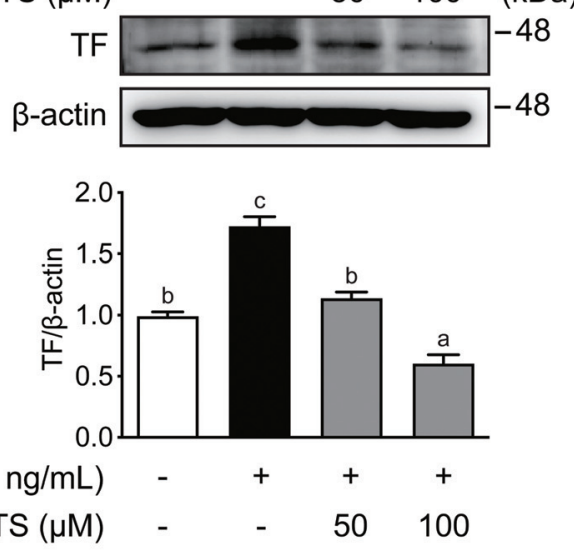

D
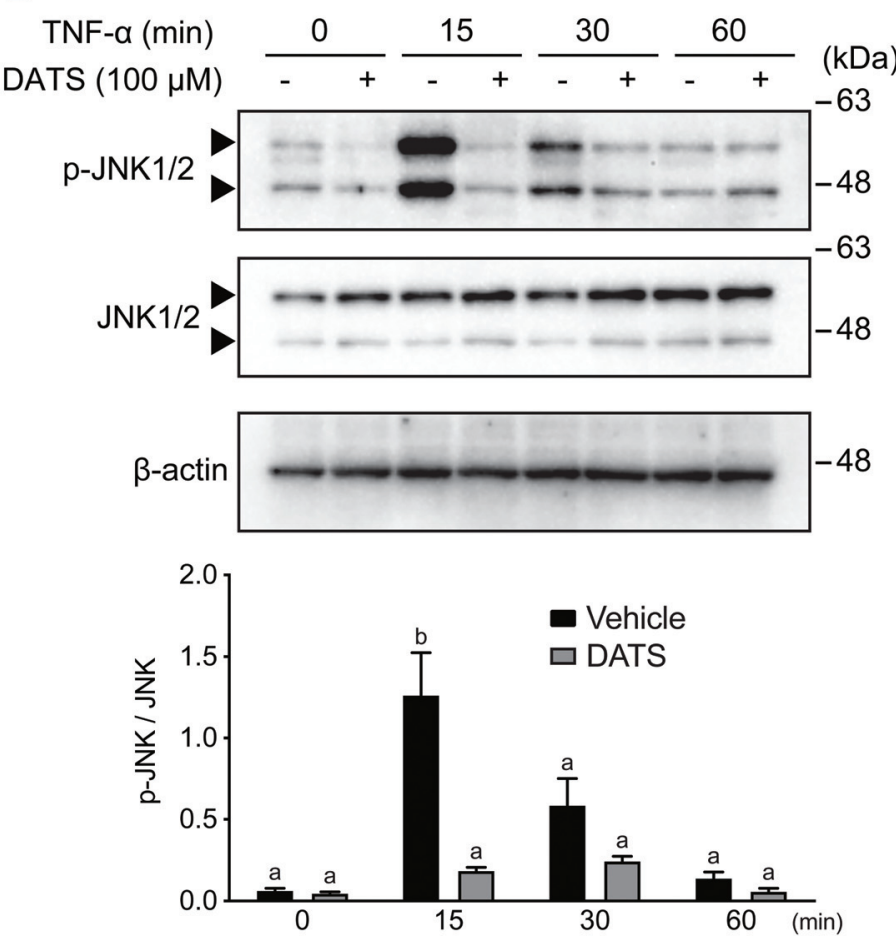

E
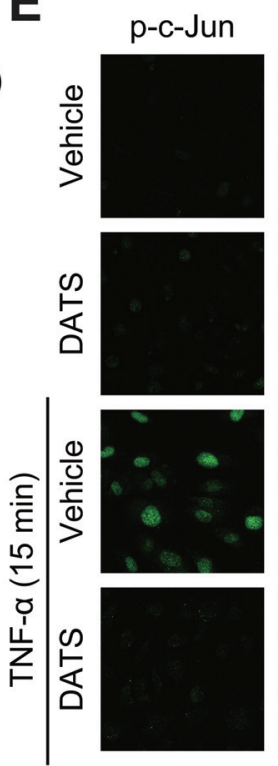

- Vehicle $\square$ DATS

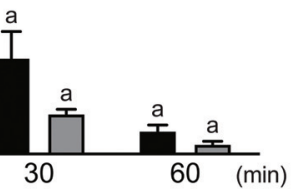

DAPI
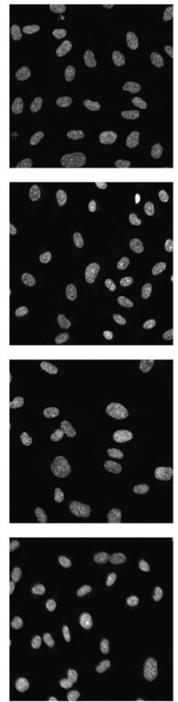

Merge
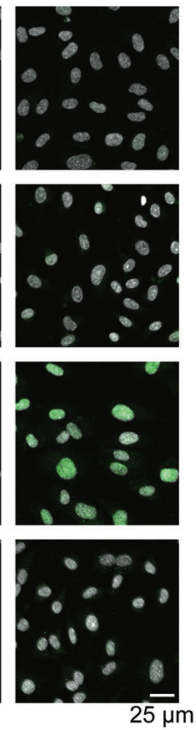

Fig. 2 DATS inhibits TNF- $\alpha$-induced TF mRNA and protein expression in HUVECs. (A) HUVECs were pre-treated with DATS or vehicle for 30 min prior to TNF- $\alpha$ treatment. After incubation with TNF- $\alpha$ for 2-8 h, the cells were harvested and measured for TF mRNA levels using real-time PCR. (B) After incubation with TNF- $\alpha$ for 2-8 h, the cells were harvested, and TF protein levels were measured by western blotting. TF levels were analysed quantitatively using ImageJ software and normalised to those of $\beta$-actin. (C) HUVECs were pre-treated with DATS (50 or $100 \mu \mathrm{M})$ or vehicle for $30 \mathrm{~min}$, followed by treatment with TNF- $\alpha$ for $4 \mathrm{~h}$. TF protein levels were measured by western blotting as described above. (D) Phosphorylation of JNK1/2 in the DATS-treated HUVECs was determined by western blotting. $p$-JNK levels were analysed quantitatively as described above. Mean \pm SE $(n=3)$. Groups labelled with the same superscripts are not significantly difference from each other. Groups labelled with different superscripts are significantly different from each other. (E) HUVECs were pre-treated with DATS or vehicle for 30 min, followed by treatment with TNF- $\alpha$ for 15 min. The images of phosphorylated-c-Jun localisation in nuclei were obtained by confocal microscopy. 

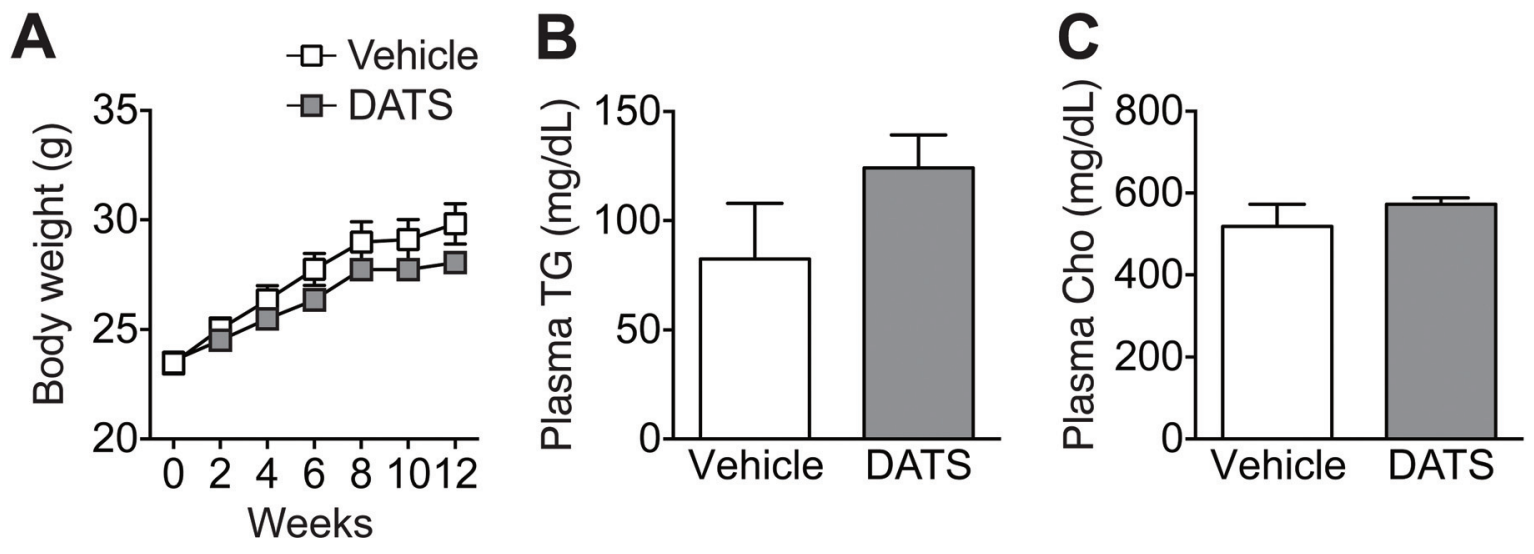

D
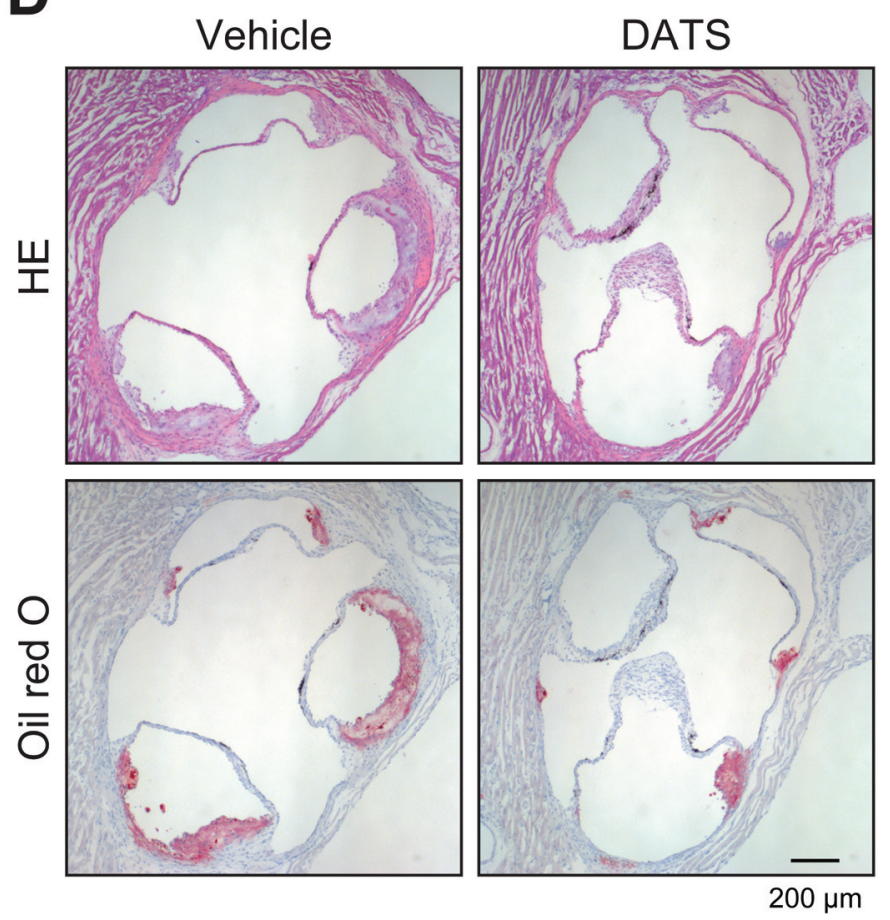

E

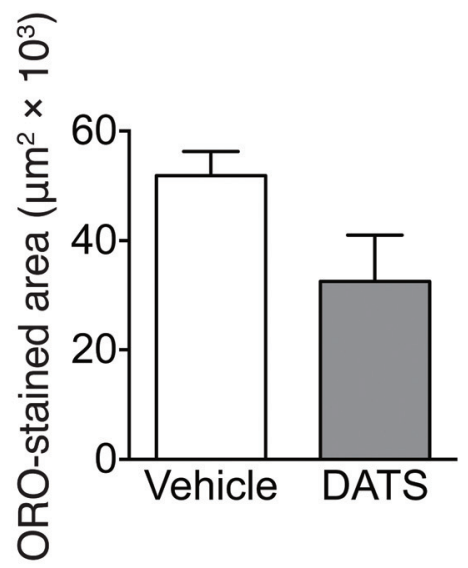

Fig. 3 DATS ameliorated arteriosclerotic lesions in ApoE-deficient mice. ApoE-deficient mice were treated with DATS or vehicle every other day for 12 weeks. (A) Body weight gain. (B) Plasma triacylglycerol concentration. (C) Plasma cholesterol concentration. (D) Serial cryosections of the tricuspid valve were stained with haematoxylin and eosin (HE) (upper panels) or Oil Red O (lower panels). (E) The area stained with Oil Red O was quantified as the lesion size using ImageJ software. Mean \pm SE. Vehicle, $n=4$; DATS, $n=5$.

\section{Discussion}

TF is a key initiator of the extrinsic coagulation pathway. TF is involved not only in physiological haemostasis but also in pathological processes during cardiovascular events via its effects on arteriosclerosis. Therefore, the regulation of $\mathrm{TF}$ expression and activity is an important strategy for the prevention of thromboembolism triggered by lifestyle-related diseases. ${ }^{34}$ Various dietary factors have been shown to exert preventive effects on lifestyle-related diseases. In this study, we examined the effects of DATS on extrinsic coagulation initiated by TF. DATS has potent anti-platelet activity, showing promise as an anticoagulant.
To examine the effects of DATS on TF activity, we used a clotting assay system with PMA-stimulated TF-expressing HL60 cells. $^{8,18,19}$ DATS delayed clotting time in a dose-dependent manner (Fig. 1B). The effect of NEM on clot formation in comparison with that of DATS was also examined. NEM delayed clotting time in a manner similar to DATS (Fig. 1C). NEM is a Michael acceptor in the Michael reaction, meaning that it adds nucleophiles, such as the thiolate anion, and is commonly used to protect cysteine thiol groups in proteins against oxidation. $^{35}$ Taken together, these data suggest that modification of cysteine thiols in the TF molecule is an important mechanism underlying the inhibition of TF activity by both DATS and NEM. 

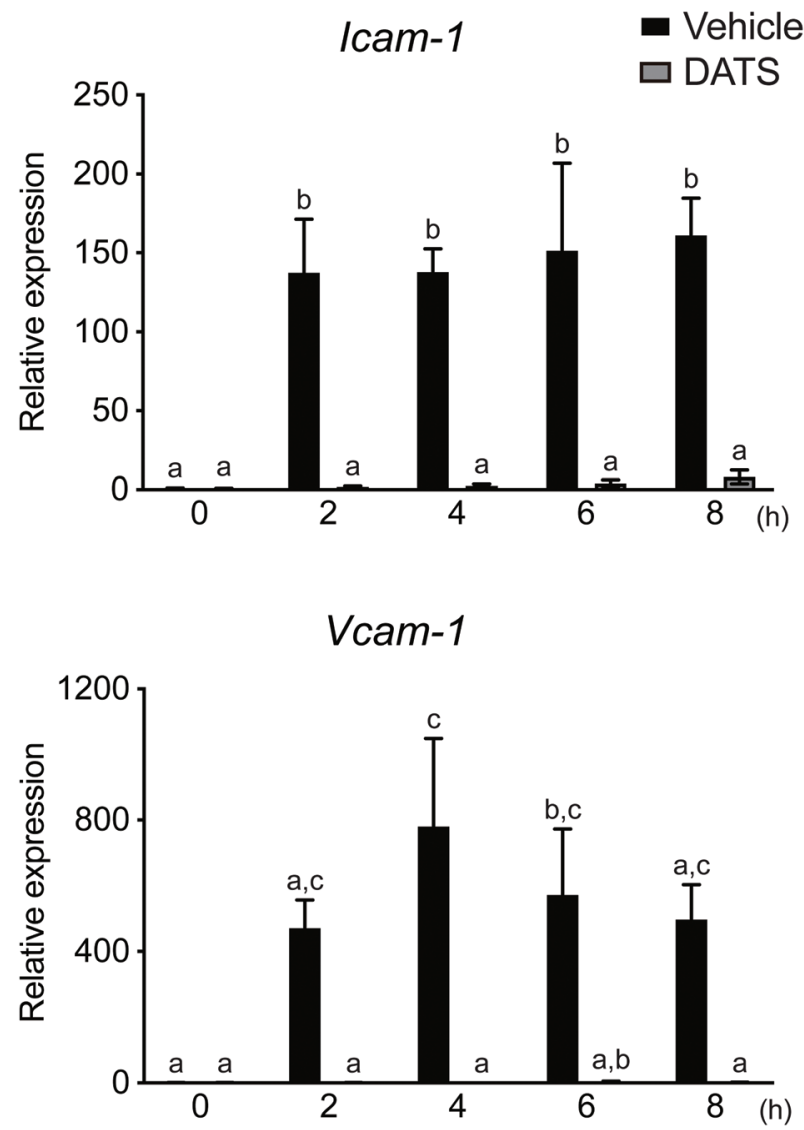

Fig. 4 DATS inhibits TNF- $\alpha$-induced mRNA expression of Icam-1 and Vcam-1 in HUVECs. HUVECs were pre-treated with DATS $(100 \mu \mathrm{M})$ or vehicle for 30 min prior to TNF- $\alpha$ treatment. After incubation with TNF- $\alpha$ for 2-8 $h$, the cells were harvested and Icam-1 and Vcam-1 mRNA levels were measured by real-time PCR. Mean \pm SE $(n=3)$. Groups labelled with the same superscripts are not significantly different from each other. Groups labelled with different superscripts are significantly different from each other.

We next investigated the regulation of TF by DATS in vascular endothelial cells. Normal vascular endothelial cells do not express TF in vitro; however, inflammatory mediators, such as TNF- $\alpha$ and lipopolysaccharide, induce TF expression. ${ }^{5,7}$ In addition, it has been reported that plasma TF concentrations are higher in patients with obesity, dyslipidaemia and type 2 diabetes than in healthy subjects. ${ }^{36,37}$ It has also been reported that the concentrations of plasma TNF- $\alpha$ and microparticles containing TF in blood are elevated in patients with type 2 diabetes. $^{38}$ Lifestyle-related diseases, including obesity, dyslipidaemia and type 2 diabetes, are strongly related to the development of arteriosclerosis. ${ }^{39,40}$ Therefore, the suppression of soluble TF levels in blood may contribute to the prevention of arteriosclerosis associated with these diseases.

$\mathrm{TF}$ production is regulated at the gene expression level. ${ }^{41,42}$ Incubation of HUVECs with TNF- $\alpha$ induced TF mRNA expression and subsequent TF protein synthesis (Fig. 2A and B). Both TF mRNA and protein expression levels were increased 2-4 h after TNF- $\alpha$ treatment (Fig. 2A and B). These increases were inhibited by pre-treatment of HUVECs with DATS $30 \mathrm{~min}$ prior to TNF- $\alpha$ treatment (Fig. $2 \mathrm{~A}$ and C). TNF- $\alpha$ induces TF expression via JNK/c-Jun signalling in HUVECs. ${ }^{10}$ In our study, phosphorylation of JNK and localisation of phosphorylated c-Jun in the nucleus induced by TNF- $\alpha$ were suppressed by DATS (Fig. 2D). It has been reported that TNF$\alpha$-induced TF mRNA expression in vascular endothelial cells was suppressed by the inhibition of JNK and p38. ${ }^{10}$ Therefore, inhibition of the JNK pathway by DATS may inhibit the induction of TF by TNF- $\alpha$. DATS not only inhibited TF activity, but also TF mRNA and protein expression in vitro.

As described previously, the plasma TF concentration is correlated with arteriosclerosis. ${ }^{15,43}$ Based on these findings, we further explored the effects of DATS on arteriosclerosis in vivo. We evaluated ApoE-deficient mice to examine the effects of DATS on arteriosclerosis because arteriosclerosis is rarely observed in normal wild-type mice on a standard diet. It has been shown that blood cholesterol concentrations are higher in ApoE-deficient mice than wild-type mice, specifically due to the ApoE deficiency, and aortic and coronary artery plaque formation occur at 3-4 months of age. ${ }^{44}$ In addition, a high-fat diet promotes plaque formation in comparison to feeding normal chow. ${ }^{45}$ Therefore, the mice were fed normal chow, which can lead to mild arteriosclerosis in ApoE-deficient mice.

We evaluated chow-fed ApoE-deficient mice to determine the effects of DATS administered orally every other day on plaque formation in cases of mild arteriosclerosis. Representative sections stained with Oil Red O revealed suppressed plaque formation in the tricuspid valve in the DATS treatment group compared with the vehicle group (Fig. 3D); however, the results were not statistically significant (Fig. 3E). DATS did not exert any effect on blood cholesterol concentration in ApoE-deficient mice (Fig. 3C), suggesting that DATS suppresses plaque formation without regulating cholesterol metabolism.

The inflammatory response of macrophages is closely involved in atherogenesis. ${ }^{46}$ In human atherogenesis, macrophage infiltration is observed during lipid accumulation, and foam cells can be detected during the pathological intimal thickening stage. ${ }^{47}$ In a previous study, macrophage infiltration was observed together with promotion of lipid accumulation in the tricuspid valve after 6-9 weeks of feeding with a high-fat diet in low-density lipoprotein receptor-deficient mice. ${ }^{48}$ However, in this previous study, the TF and macrophage markers, CD11b and F4/80, were undetectable in both the vehicle and DATS treatment groups according to immunostaining of the tricuspid valve, whereas plaques were observed in our ApoE-deficient mice (data not shown). This may have been due to observation of early-stage atherogenesis without macrophage infiltration, as our study involved chow-fed mice observed over a 12-week period. In addition, the inflammatory response in vascular endothelial cells is involved in the formation of plaque by stimulation of the interaction between leukocytes and activated platelets via adhesion molecules. In particular, inflammatory vascular endothelial cells activated by inflammatory mediators, such as $\mathrm{TNF}-\alpha$, express Icam- 1 and 
Vcam-1. ${ }^{49}$ In this study, the levels of Icam-1 and Vcam1 mRNA expression were increased by TNF- $\alpha$ in HUVECs, and pre-treatment with DATS markedly suppressed their expression (Fig. 4). It has been reported that plaque formation was suppressed in ApoE, P-selectin, Icam-1 and E-selectin-deficient mice. ${ }^{50}$ It has also been reported that Vcam-1 participates in plaque formation to a greater extent than Icam-1. ${ }^{51}$ Therefore, DATS may suppress plaque formation by inhibiting the expression of adhesion molecules in inflammatory vascular endothelial cells.

Recently, we reported that DATS strongly inhibits platelet aggregation because of its high reactivity with $\mathrm{SH}$ groups by suppressing cell signalling and cell surface activation induced by thrombin. ${ }^{28}$ Although DATS also has potent anti-platelet activity, bleeding tendency was not observed in the tail bleeding assay in mice. ${ }^{28}$ We also reported inhibition of platelet aggregation by methyl allyl trisulphide via inhibition of hydroperoxidase activity and subsequent thromboxane $\mathrm{A}_{2}$ production, which led to inhibition of clot formation in an ex vivo thrombosis model. ${ }^{27,52}$

In summary, we demonstrated that DATS inhibits TF activity and mRNA and protein expression in vitro. Furthermore, DATS tended to ameliorate plaque formation in ApoE-deficient mice in vivo. DATS may serve as a dietary constituent with beneficial effects on vascular diseases, including atherosclerosis, and as an antithrombotic agent via its effects on the extrinsic coagulation pathway.

\section{Conclusions}

To our knowledge, this is the first study to demonstrate that DATS inhibits TF expression in endothelial cells as well as TF protein activity. The atherosclerotic lesion size also tended to be decreased in ApoE-deficient mice treated with DATS. Furthermore, we demonstrated that DATS exhibits potent antiplatelet activity. Thromboembolism is caused by platelets (primary thrombus formation) and subsequent clot formation via the coagulation cascade (secondary thrombus formation). Garlic is a promising food with anti-thrombotic function, which can suppress both primary and secondary clot formation.

\section{Author contributions}

Sachiko Okue: Conceptualisation, data curation, investigation, methodology, writing-original draft; Manami Yaguchi: Conceptualisation, data curation, investigation, methodology; Atsushi Miura: Data curation, investigation, methodology, validation, visualisation, writing-original draft; Yori OzakiMasuzawa: Writing-review \& editing; Takashi Hosono: Conceptualisation, investigation, methodology, project administration, validation, writing-review \& editing; Taiichiro Seki: Conceptualisation, funding acquisition, investigation, project administration, validation, writing-review \& editing.

\section{Conflicts of interest}

The authors report no potential conflicts of interest.

\section{Acknowledgements}

This work was supported by the Japan Society for the Promotion of Science KAKENHI (grant numbers 19H02914, 25292077 and 23780145).

\section{References}

1 B. Osterud and E. Bjorklid, Sources of tissue factor, Semin. Thromb. Hemostasis, 2006, 32, 11-23.

2 B. Dahlbäck, Blood coagulation, Lancet, 2000, 355, 16271632.

3 D. Saha, S. Saha, E. G. Sergeeva, Z. I. Ionova and A. V. Gorbach, Tissue factor and atherothrombosis, Curr. Pharm. Des., 2015, 21, 1152-1157.

4 Q. Choi, K. H. Hong, J. E. Kim and H. K. Kim, Changes in plasma levels of natural anticoagulants in disseminated intravascular coagulation: high prognostic value of antithrombin and protein $\mathrm{C}$ in patients with underlying sepsis or severe infection, Ann. Lab. Med., 2014, 34, 85-91.

5 G. C. Parry and N. Mackman, Transcriptional regulation of tissue factor expression in human endothelial cells, Arterioscler., Thromb., Vasc. Biol., 1995, 15, 612-621.

6 M. Colucci, G. Balconi, R. Lorenzet, A. Pietra, D. Locati, M. B. Donati and N. Semeraro, Cultured human endothelial cells generate tissue factor in response to endotoxin, J. Clin. Invest., 1983, 71, 1893-1896.

7 P. Houston, M. C. Dickson, V. Ludbrook, B. White, J. L. Schwachtgen, J. H. McVey, N. Mackman, J. M. Reese, D. G. Gorman, C. Campbell and M. Braddock, Fluid shear stress induction of the tissue factor promoter in vitro and in vivo is mediated by Egr-1, Arterioscler., Thromb., Vasc. Biol., 1999, 19, 281-289.

8 M. Z. Cui, G. C. Parry, T. S. Edgington and N. Mackman, Regulation of tissue factor gene expression in epithelial cells. Induction by serum and phorbol 12-myristate 13-acetate, Arterioscler. Thromb., 1994, 14, 807-814.

9 J. Steffel, A. Akhmedov, H. Greutert, T. F. Lüscher and F. C. Tanner, Histamine induces tissue factor expression: implications for acute coronary syndromes, Circulation, 2005, 112, 341-349.

10 Y. Liu, K. Pelekanakis and M. J. Woolkalis, Thrombin and tumor necrosis factor $\alpha$ synergistically stimulate tissue factor expression in human endothelial cells: regulation through c-Fos and c-Jun, J. Biol. Chem., 2004, 279, 3614236147

11 Y. Liang, X. Li, X. Zhang, Z. Li, L. Wang, Y. Sun, Z. Liu and $\mathrm{X}$. Ma, Elevated levels of plasma TNF- $\alpha$ are associated with microvascular endothelial dysfunction in patients with sepsis through activating the NF- $\mathrm{KB}$ and p38 mitogen-acti- 
vated protein kinase in endothelial cells, Shock, 2014, 41, 275-281.

12 Y. Liu, L. Zhang, C. Wang, S. Roy and J. Shen, Purinergic P2Y2 Receptor Control of Tissue Factor Transcription in Human Coronary Artery Endothelial Cells: NEW AP-1 TRANSCRIPTION FACTOR SITE AND NEGATIVE REGULATOR, J. Biol. Chem., 2016, 291, 1553-1563.

13 S. C. Landers, M. Gupta and J. C. Lewis, Ultrastructural localization of tissue factor on monocyte-derived macrophages and macrophage foam cells associated with atherosclerotic lesions, Virchows Arch., 1994, 425, 49-54.

14 J. N. Wilcox, K. M. Smith, S. M. Schwartz and D. Gordon, Localization of tissue factor in the normal vessel wall and in the atherosclerotic plaque, Proc. Natl. Acad. Sci. U. S. A., 1989, 86, 2839-2843.

15 J. Steffel, T. F. Lüscher and F. C. Tanner, Tissue factor in cardiovascular diseases: molecular mechanisms and clinical implications, Circulation, 2006, 113, 722-731.

16 J. I. Zwicker, C. C. Trenor, B. C. Furie and B. Furie, Tissue factor-bearing microparticles and thrombus formation, Arterioscler., Thromb., Vasc. Biol., 2011, 31, 728-733.

17 H. Kothari, R. C. Nayak, L. V. Rao and U. R. Pendurthi, Cystine 186-cystine 209 disulfide bond is not essential for the procoagulant activity of tissue factor or for its deencryption, Blood, 2010, 115, 4273-4283.

18 V. M. Chen, J. Ahamed, H. H. Versteeg, M. C. Berndt, W. Ruf and P. J. Hogg, Evidence for activation of tissue factor by an allosteric disulfide bond, Biochemistry, 2006, 45, 12020-12028.

19 H. P. Liang, T. M. Brophy and P. J. Hogg, Redox properties of the tissue factor Cys186-Cys209 disulfide bond, Biochem. J., 2011, 437, 455-460.

20 T. Seki, T. Hosono and Y. Ozaki-Masuzawa, in Chemistry of Korean Foods and Beverages, ed. C. H. Do, A. M. Rimando and Y. Kim, American Chemical Society, Washington, DC, 2019, ch. 4, pp. 43-55.

21 K. Ried and P. Fakler, Potential of garlic (Allium sativum) in lowering high blood pressure: mechanisms of action and clinical relevance, Integr. Blood Pressure Control, 2014, 7, 71-82.

22 K. Ried, O. R. Frank and N. P. Stocks, Aged garlic extract reduces blood pressure in hypertensives: a dose-response trial, Eur. J. Clin. Nutr., 2013, 67, 64-70.

23 Y. Kagawa, Y. Ozaki-Masuzawa, T. Hosono and T. Seki, Garlic oil suppresses high-fat diet induced obesity in rats through the upregulation of UCP-1 and the enhancement of energy expenditure, Exp. Ther. Med., 2020, 19, 15361540.

24 A. Miura, A. Ikeda, M. Abe, K. Seo, T. Watanabe, Y. OzakiMasuzawa, T. Hosono and T. Seki, Diallyl Trisulfide Prevents Obesity and Decreases miRNA-335 Expression in Adipose Tissue in a Diet-Induced Obesity Rat Model, Mol. Nutr. Food Res., 2021, 65, e2001199.

25 C. Yang, L. Li, L. Yang, H. Lu, S. Wang and G. Sun, Antiobesity and Hypolipidemic effects of garlic oil and onion oil in rats fed a high-fat diet, Nutr. Metab., 2018, 15, 43.
26 A. Bordia, S. K. Verma, A. K. Vyas, B. L. Khabya, A. S. Rathore, N. Bhu and H. K. Bedi, Effect of essential oil of onion and garlic on experimental atherosclerosis in rabbits, Atherosclerosis, 1977, 26, 379-386.

27 T. Ariga, S. Oshiba and T. Tamada, Platelet aggregation inhibitor in garlic, Lancet, 1981, 1, 150-151.

28 T. Hosono, A. Sato, N. Nakaguchi, Y. Ozaki-Masuzawa and T. Seki, Diallyl Trisulfide Inhibits Platelet Aggregation through the Modification of Sulfhydryl Groups, J. Agric. Food Chem., 2020, 68, 1571-1578.

29 J. Borlinghaus, F. Albrecht, M. C. Gruhlke, I. D. Nwachukwu and A. J. Slusarenko, Allicin: chemistry and biological properties, Molecules, 2014, 19, 1259112618.

30 D. Lievens and P. von Hundelshausen, Platelets in atherosclerosis, Thromb. Haemostasis, 2011, 106, 827-838.

31 K. J. Livak and T. D. Schmittgen, Analysis of relative gene expression data using real-time quantitative PCR and the 2(-Delta C(T)) Method, Methods, 2001, 25, 402-408.

32 T. Okamoto and K. Suzuki, The Role of Gap JunctionMediated Endothelial Cell-Cell Interaction in the Crosstalk between Inflammation and Blood Coagulation, Int. J. Mol. Sci., 2017, 18, 2254.

33 R. W. Mahley, Apolipoprotein E: cholesterol transport protein with expanding role in cell biology, Science, 1988, 240, 622-630.

34 T. Seki and T. Hosono, Prevention of Cardiovascular Diseases by Garlic-Derived Sulfur Compounds, J. Nutr. Sci. Vitaminol., 2015, 61 Suppl, S83-S85.

35 J. R. Winther and C. Thorpe, Quantification of thiols and disulfides, Biochim. Biophys. Acta, 2014, 1840, 838-846.

36 A. Sambola, J. Osende, J. Hathcock, M. Degen, Y. Nemerson, V. Fuster, J. Crandall and J. J. Badimon, Role of risk factors in the modulation of tissue factor activity and blood thrombogenicity, Circulation, 2003, 107, 973-977.

37 R. S. El-Hagracy, G. M. Kamal, I. M. Sabry, A. A. Saad, N. F. Abou El Ezz and H. A. Nasr, Tissue factor, tissue factor pathway inhibitor and factor VII activity in cardiovascular complicated type 2 diabetes mellitus, Oman Med. J., 2010, 25, 173-178.

38 M. Diamant, R. Nieuwland, R. F. Pablo, A. Sturk, J. W. Smit and J. K. Radder, Elevated numbers of tissue-factor exposing microparticles correlate with components of the metabolic syndrome in uncomplicated type 2 diabetes mellitus, Circulation, 2002, 106, 2442-2447.

39 L. E. Wagenknecht, D. Zaccaro, M. A. Espeland, A. J. Karter, D. H. O'Leary and S. M. Haffner, Diabetes and progression of carotid atherosclerosis: the insulin resistance atherosclerosis study, Arterioscler., Thromb., Vasc. Biol., 2003, 23, 1035-1041.

40 H. C. McGill, C. A. McMahan, E. E. Herderick, A. W. Zieske, G. T. Malcom, R. E. Tracy, J. P. Strong and Pathobiological Determinants of Atherosclerosis in Youth (PDAY) Research Group, Obesity accelerates the progression of coronary atherosclerosis in young men, Circulation, 2002, 105, 27122718. 
41 K. Brand, B. J. Fowler, T. S. Edgington and N. Mackman, Tissue factor mRNA in THP-1 monocytic cells is regulated at both transcriptional and posttranscriptional levels in response to lipopolysaccharide, Mol. Cell. Biol., 1991, 11, 4732-4738.

42 S. M. Ahern, T. Miyata and J. E. Sadler, Regulation of human tissue factor expression by mRNA turnover, J. Biol. Chem., 1993, 268, 2154-2159.

43 I. Müller, A. Klocke, M. Alex, M. Kotzsch, T. Luther, E. Morgenstern, S. Zieseniss, S. Zahler, K. Preissner and B. Engelmann, Intravascular tissue factor initiates coagulation via circulating microvesicles and platelets, FASEB J., 2003, 17, 476-478.

44 A. S. Plump, J. D. Smith, T. Hayek, K. Aalto-Setälä, A. Walsh, J. G. Verstuyft, E. M. Rubin and J. L. Breslow, Severe hypercholesterolemia and atherosclerosis in apolipoprotein E-deficient mice created by homologous recombination in ES cells, Cell, 1992, 71, 343-353.

45 R. Wang, Y. Zhang, L. Xu, Y. Lin, X. Yang, L. Bai, Y. Chen, S. Zhao, J. Fan, X. Cheng and E. Liu, Protein inhibitor of activated STAT3 suppresses oxidized LDL-induced cell responses during atherosclerosis in apolipoprotein E-deficient mice, Sci. Rep., 2016, 6, 36790.

46 A. J. Lusis, Atherosclerosis, Nature, 2000, 407, 233-241.

47 Y. Nakashima, H. Fujii, S. Sumiyoshi, T. N. Wight and K. Sueishi, Early human atherosclerosis: accumulation of lipid and proteoglycans in intimal thickenings followed by macrophage infiltration, Arterioscler., Thromb., Vasc. Biol., 2007, 27, 1159-1165.

48 D. Ye, Y. Zhao, R. B. Hildebrand, R. R. Singaraja, M. R. Hayden, T. J. Van Berkel and M. Van Eck, The dynamics of macrophage infiltration into the arterial wall during atherosclerotic lesion development in low-density lipoprotein receptor knockout mice, Am. J. Pathol., 2011, 178, 413-422.

49 F. Zhang, W. Yu, J. L. Hargrove, P. Greenspan, R. G. Dean, E. W. Taylor and D. K. Hartle, Inhibition of TNF- $\alpha$ induced ICAM-1, VCAM-1 and E-selectin expression by selenium, Atherosclerosis, 2002, 161, 381-386.

50 R. G. Collins, R. Velji, N. V. Guevara, M. J. Hicks, L. Chan and A. L. Beaudet, P-Selectin or intercellular adhesion molecule (ICAM)-1 deficiency substantially protects against atherosclerosis in apolipoprotein E-deficient mice, J. Exp. Med., 2000, 191, 189-194.

51 M. I. Cybulsky, K. Iiyama, H. Li, S. Zhu, M. Chen, M. Iiyama, V. Davis, J. C. Gutierrez-Ramos, P. W. Connelly and D. S. Milstone, A major role for VCAM-1, but not ICAM-1, in early atherosclerosis, J. Clin. Invest., 2001, 107, 1255-1262.

52 T. Ariga, K. Tsuj, T. Seki, T. Moritomo and J. I. Yamamoto, Antithrombotic and antineoplastic effects of phyto- organosulfur compounds, BioFactors, 2000, 13, 251-255. 